\title{
Expressivity in Chain-Based Modal Logics
}

\author{
Michel Marti · George Metcalfe
}

Received: date / Accepted: date

\begin{abstract}
We investigate the expressivity of many-valued modal logics based on an algebraic structure with a complete linearly ordered lattice reduct. Necessary and sufficient algebraic conditions for admitting a suitable Hennessy-Milner property are established for classes of image-finite and (appropriately defined) modally saturated models. Full characterizations are obtained for many-valued modal logics based on complete BL-chains that are finite or have the real unit interval $[0,1]$ as a lattice reduct, including Łukasiewicz, Gödel, and product modal logics. ${ }^{1}$
\end{abstract}

\section{Introduction}

Many-valued modal logics generalize the Kripke semantics of classical modal logic to incorporate a local many-valued semantics based on an algebra with a complete lattice reduct, where the accessibility relation may be crisp (Boolean-valued) or take values in the algebra itself (see, e.g., $[13,14,26,5]$ ). Such logics can be designed to model modal notions such as necessity, belief, and spatio-temporal relations in the presence of uncertainty, possibility, or vagueness, spanning, e.g., fuzzy belief [15], fuzzy similarity measures [16], spatial reasoning with vague predicates [27], and many-valued tense logics $[19,10]$. Many-valued multi-modal logics also provide a basis, as in the classical setting, for defining fuzzy description logics (see, e.g., [18, $28,4]$ ). More generally, many-valued modal logics provide a first foray, similarly to

Supported by Swiss National Science Foundation grant 200021_165850.

\section{Marti}

Institute of Computer Science, University of Bern, Neubrückstrasse 10, CH-3012 Bern, Switzerland

E-mail: mmarti@inf.unibe.ch

G. Metcalfe

Mathematical Institute, University of Bern, Sidlerstrasse 5, CH-3012 Bern, Switzerland

E-mail: george.metcalfe@math.unibe.ch

1 Preliminary results from this work were reported in the proceedings of AiML 2014 [21]. 
the classical case, into investigating useful and computationally feasible fragments of corresponding many-valued first-order logics.

The study of many-valued modal logics is still very much in its infancy and has focussed to date mostly on issues of axiomatization, decidability, and complexity. Other topics from the rich theory of classical modal logic have not as yet received much attention. In particular, the question of the expressivity of many-valued modal logics has remained, until very recently (see [21,2] and the remarks in Section 6), largely unexplored. In the classical setting, Van Benthem's theorem demonstrates that the modal logic $\mathrm{K}$ may be viewed as the bisimulation-invariant fragment of first-order logic, and it may be asked if similar results hold in a many-valued context. To address this challenging question, we ask first whether analogues of the Hennessy-Milner property - modal equivalence coinciding with bisimilarity - hold for classes of models for a many-valued modal logic, focussing in particular on the class of imagefinite models (i.e., where each state has finitely many successors) and a broader class of modally saturated models. Modal equivalence between two states means here that each formula takes the same value in both states, while the definition of a bisimulation matches the classical notion except that variables take the same value in bisimilar states.

We restrict our attention in this paper to crisp Kripke frames and assume that the underlying algebra has a complete chain (linearly ordered lattice) reduct. This framework spans many-valued modal logics defined over a complete MTL-chain (integral commutative residuated chain): in particular, the families of Gödel, Łukasiewicz, and product modal logics studied in [7,23,8,6], [17,20,11], and [29], respectively. More concretely, in Section 2, we define a many-valued modal logic $\mathrm{K}(\mathbf{A})^{\mathrm{C}}$ based on a single algebra $\mathbf{A}$ with a complete chain reduct and provide necessary and sufficient algebraic conditions on $\mathbf{A}$ for the classes of image-finite models (Section 3) and suitably defined modally saturated models (Section 4) to admit the Hennessy-Milner property.

In Section 5, we obtain full characterizations when $\mathbf{A}$ is a BL-chain (a divisible MTL-chain) that is finite or has the real unit interval $[0,1]$ as a lattice reduct. In both cases, the class of image-finite models admits the Hennessy-Milner property precisely when $\mathbf{A}$ is an MV-chain (a BL-chain with an involutive negation) or the ordinal sum of two MV-chains. In particular, the class of image-finite models for each Łukasiewicz modal logic and the three-valued Gödel modal logic admits the Hennessy-Milner property, but this does not hold for product modal logic or Gödel modal logics with more than three truth values. For the class of modally saturated models to admit the Hennessy-Milner property, the BL-chain A must also be finite.

\section{Many-Valued Kripke Models}

Let $\mathcal{L}$ be a finite algebraic language that includes the binary operation symbols $\wedge$ and $\vee$ and the constant symbols $\perp$ and $T$. An algebraic structure $\mathbf{A}$ for $\mathcal{L}$ will be called chain-based if the reduct $\left\langle A, \wedge^{\mathbf{A}}, \vee^{\mathbf{A}}, \perp^{\mathbf{A}}, \top^{\mathbf{A}}\right\rangle$ is a complete bounded chain; i.e., the order defined by $a \leq^{\mathbf{A}} b: \Leftrightarrow a \wedge^{\mathbf{A}} b=a$ for $a, b \in A$ is linear, and $\wedge^{\mathbf{A}} B$ and 
$\bigvee^{\mathbf{A}} B$ exist in $A$ for all $B \subseteq A$. In what follows, we omit the superscript $\mathbf{A}$ when the algebra or corresponding order is clear from the context.

Example 1 We will pay particular attention to chain-based algebras that arise in the study of substructural and many-valued logics (see, e.g., [17,24,25,5]). An MTLalgebra — where MTL stands for monoidal t-norm logic — is an algebraic structure

$$
\mathbf{A}=\langle A, \wedge, \vee, \cdot, \rightarrow, \perp, \top\rangle
$$

such that $\langle A, \wedge, \vee, \perp, \top\rangle$ is a bounded lattice, $\langle A, \cdot, \top\rangle$ is a commutative monoid, and for all $a, b, c \in A$,

$$
a \cdot b \leq c \quad \Longleftrightarrow \quad a \leq b \rightarrow c .
$$

For $a \in A$, we also fix $\neg a:=a \rightarrow \perp$ and $a \leftrightarrow b:=(a \rightarrow b) \wedge(b \rightarrow a)$, and define inductively $a^{0}:=\top$ and $a^{n+1}:=a \cdot a^{n}$ for $n \in \mathbb{N}$.

An MTL-algebra A is divisible and a BL-algebra - where BL stands for basic logic - if whenever $a \leq b$ in $\mathbf{A}$, there exists $c$ in $A$ such that $b \cdot c=a$, or, equivalently, A satisfies the identity $x \cdot(x \rightarrow y) \approx y \cdot(y \rightarrow x)$. If the lattice reduct of an MTLalgebra or BL-algebra $\mathbf{A}$ is linearly ordered, then $\mathbf{A}$ is an MTL-chain or a BL-chain, respectively. In particular, if the lattice reduct of $\mathbf{A}$ is the real unit interval $[0,1]$ with the usual order, then $\cdot$ is a left-continuous t-norm with unit $\top=1$ and residual $\rightarrow$, and $\mathbf{A}$ is a standard MTL-chain; if - is also continuous, then $\mathbf{A}$ is a standard BLchain. Such algebras provide standard semantics for Łukasiewicz logic, Gödel logic, and product logic when · is the Łukasiewicz $t$-norm $\max (0, x+y-1)$, the minimum (Gödel) $t$-norm $\min (x, y)$, or the product $t$-norm $x y$ (multiplication), respectively.

Let $\mathcal{L}_{\square \diamond}$ be the language $\mathcal{L}$ extended with unary connectives $\square$ and $\diamond$. The set of formulas $\mathrm{Fm}_{\square \diamond}$ of this language, with arbitrary members denoted by $\varphi, \psi, \ldots$, is defined inductively over a countably infinite set Var of propositional variables, with arbitrary members denoted by $p, q, \ldots$. The set of propositional formulas will be denoted by Fm. Subformulas are defined as usual, the length $\ell(\varphi)$ of $\varphi \in \mathrm{Fm}_{\square \diamond}$ is the number of symbols occurring in $\varphi$, and we write $\varphi\left(p_{1}, \ldots, p_{n}\right)$ to denote that the variables occurring in $\varphi$ are among $p_{1}, \ldots, p_{n}$.

A frame is an ordered pair $\langle W, R\rangle$, where $W$ is a non-empty set of states and $R \subseteq W \times W$ is a binary accessibility relation on $W$. As usual, we write $R u v$ or $R u v=1$ to denote $\langle u, v\rangle \in R$ and $R u v=0$ to denote $\langle u, v\rangle \notin R$. For any $u \in W$, we define $R[u]:=\{v \in W \mid R u v\}$.

Now let $\mathbf{A}$ be a chain-based algebra. $\mathrm{A} \mathrm{K}(\mathbf{A})^{\mathrm{C}}$-model $^{2}$ is a triple $\mathfrak{M}=\langle W, R, V\rangle$, where $\langle W, R\rangle$ is a frame and $V: \operatorname{Var} \times \mathrm{W} \rightarrow \mathrm{A}$ is a mapping, called a valuation, extended to $V: \mathrm{Fm}_{\square \diamond} \times \mathrm{W} \rightarrow \mathrm{A}$ by

$$
V\left(\star\left(\varphi_{1}, \ldots, \varphi_{n}\right), w\right)=\star^{\mathbf{A}}\left(V\left(\varphi_{1}, w\right), \ldots, V\left(\varphi_{n}, w\right)\right)
$$

\footnotetext{
2 The superscript ${ }^{C}$ denotes that the frame is "crisp"; more generally, a $\mathrm{K}(\mathbf{A})$-model is defined for frames equipped with a "many-valued" accessibility relation $R: W \times W \rightarrow A$ and the conditions for $\square, \diamond$ are revised accordingly (see [5] for details).
} 
for each $n$-ary operation symbol $\star$ of $\mathcal{L}$, and

$$
\begin{aligned}
& V(\square \varphi, w)=\bigwedge\{V(\varphi, v) \mid R w v\} \\
& V(\diamond \varphi, w)=\bigvee\{V(\varphi, v) \mid R w v\} .
\end{aligned}
$$

A general definition of validity of formulas in $\mathrm{K}(\mathbf{A})^{\mathrm{C}}$-models is not required for considering relationships between modal equivalence and bisimulations. Indeed, such a definition depends anyway on the intended interpretation of the logic. Nevertheless, the following example provides a standard definition for a large family of manyvalued modal logics defined over a complete MTL-chain.

Example 2 Suppose that $\mathbf{A}$ is a complete MTL-chain (see Example 1). Then we may say that $\varphi \in \mathrm{Fm}_{\square \diamond}$ is valid in a $\mathrm{K}(\mathbf{A})^{\mathrm{C}}$-model $\mathfrak{M}=\langle W, R, V\rangle$ if $V(\varphi, w)=\top$ for all $w \in W$. If $\varphi$ is valid in all $\mathrm{K}(\mathbf{A})^{\mathrm{C}}$-models, then $\varphi$ is said to be $\mathrm{K}(\mathbf{A})^{\mathrm{C}}$-valid. Logics based on $\mathrm{K}(\mathbf{A})^{\mathrm{C}}$-validity (and also $\mathrm{K}(\mathbf{A})$-validity, where the relation $R$ can take values in $\mathbf{A}$ ) have been investigated in some generality in [5], while the particular cases of Gödel, Łukasiewicz, and product modal logics have been studied in greater detail in $[7,23,8,6],[17,20,11]$, and [29], respectively.

A $\mathrm{K}(\mathbf{A})^{\mathrm{C}}$-model $\mathfrak{M}=\langle W, R, V\rangle$ is called a tree-model with root $w$ and height $n$ if $\langle W, R\rangle$ is a tree with root $w$ and height $n$. Let us also write $\left(a_{1}, \ldots, a_{n}\right) \in A^{n}$ as a vector a and given a propositional formula $\varphi\left(p_{1}, \ldots, p_{n}\right) \in \mathrm{Fm}$, let $\varphi[\mathbf{a}]$ denote the value of $\varphi$ (understood as a term function) at $\mathbf{a}$ in $\mathbf{A}$. For $\psi\left(p_{1}, \ldots, p_{n}\right) \in \mathrm{Fm}$ and $\varphi_{1}, \ldots, \varphi_{n} \in \mathrm{Fm}_{\square \diamond}$, the formula $\psi\left[\varphi_{1} / p_{1}, \ldots, \varphi_{n} / p_{n}\right]$ denotes the result of replacing all occurrences of $p_{i}$ in $\psi$ with $\varphi_{i}$ for each $i \in\{1, \ldots, n\}$. The following useful substitution lemma is proved by a straightforward induction on the length of a propositional formula.

Lemma 1 Let $\psi\left(p_{1}, \ldots, p_{n}\right) \in \mathrm{Fm}$ and $\varphi_{1}, \ldots, \varphi_{n} \in \mathrm{Fm} \square \diamond$. Then for any $\mathrm{K}(\mathbf{A})^{\mathrm{C}}$ model $\mathfrak{M}=\langle W, R, V\rangle$ and $w \in W$,

$$
V\left(\psi\left[\varphi_{1} / p_{1}, \ldots, \varphi_{n} / p_{n}\right], w\right)=\psi\left[V\left(\varphi_{1}, w\right), \ldots, V\left(\varphi_{n}, w\right)\right] .
$$

In particular, if $V\left(\varphi_{i}, w\right)=V\left(p_{i}, w\right)$ for each $i \in\{1, \ldots, n\}$, then

$$
V\left(\psi\left[\varphi_{1} / p_{1}, \ldots, \varphi_{n} / p_{n}\right], w\right)=V(\psi, w) .
$$

Consider now two $\mathrm{K}(\mathbf{A})^{\mathrm{C}}$-models $\mathfrak{M}=\langle W, R, V\rangle$ and $\mathfrak{M}^{\prime}=\left\langle W^{\prime}, R^{\prime}, V^{\prime}\right\rangle$. We will say that $w \in W$ and $w^{\prime} \in W^{\prime}$ are modally equivalent, written $w \longleftrightarrow w^{\prime}$, if $V(\varphi, w)=V^{\prime}\left(\varphi, w^{\prime}\right)$ for all $\varphi \in \mathrm{Fm}_{\square \diamond}$. The following lemma provides a useful criterion for modal equivalence when considering tree-models of height 1.

Lemma 2 Let $\mathfrak{M}=\langle W, R, V\rangle$ and $\mathfrak{M}^{\prime}=\left\langle W^{\prime}, R^{\prime}, V^{\prime}\right\rangle$ be $\mathrm{K}(\mathbf{A})^{\mathrm{C}}$-tree-models of height 1 with roots $w$ and $w^{\prime}$, respectively. Suppose that $V(p, w)=V^{\prime}\left(p, w^{\prime}\right)$ for all $p \in$ Var and $V(\square \varphi, w)=V^{\prime}\left(\square \varphi, w^{\prime}\right)$ and $V(\diamond \varphi, w)=V^{\prime}\left(\Delta \varphi, w^{\prime}\right)$ for every propositional formula $\varphi \in \mathrm{Fm}$. Then $w$ and $w^{\prime}$ are modally equivalent. 
Proof We prove the claim by induction on $\ell(\varphi)$. The base case is immediate and for the inductive step, the cases for the propositional connectives follow easily using the induction hypothesis. Suppose now that $\varphi=\square \psi$, the case $\varphi=\diamond \psi$ being very similar. Let $\psi^{*}$ be the propositional formula obtained from $\psi$ by replacing (iteratively) all subformulas of the form $\square \psi^{\prime}$ by $\top$ and all subformulas of the form $\nabla \psi^{\prime}$ by $\perp$. Then, using the fact that $\mathfrak{M}$ and $\mathfrak{M}^{\prime}$ are tree-models of height 1 , it follows by an easy induction that $V(\psi, v)=V\left(\psi^{*}, v\right)$ for all $v \in W$ such that $R w v$, and $V^{\prime}\left(\psi, v^{\prime}\right)=V^{\prime}\left(\psi^{*}, v^{\prime}\right)$ for all $v^{\prime} \in W^{\prime}$ such that $R^{\prime} w^{\prime} v^{\prime}$. But then $V(\square \psi, w)=V\left(\square \psi^{*}, w\right)=V^{\prime}\left(\square \psi^{*}, w^{\prime}\right)=V^{\prime}\left(\square \psi, w^{\prime}\right)$ as required.

A non-empty relation $Z \subseteq W \times W^{\prime}$ will be called a bisimulation between $\mathfrak{M}$ and $\mathfrak{M}^{\prime}$ if the following conditions are satisfied:

1. If $w Z w^{\prime}$, then $V(p, w)=V^{\prime}\left(p, w^{\prime}\right)$ for all $p \in \operatorname{Var}$.

2. If $w Z w^{\prime}$ and $R w v$, then there exists $v^{\prime} \in W^{\prime}$ such that $v Z v^{\prime}$ and $R^{\prime} w^{\prime} v^{\prime}$ (the forth condition).

3. If $w Z w^{\prime}$ and $R^{\prime} w^{\prime} v^{\prime}$, then there exists $v \in W$ such that $v Z v^{\prime}$ and $R w v$ (the back condition).

We say that $w \in W$ and $w^{\prime} \in W^{\prime}$ are bisimilar, written $w \equiv w^{\prime}$, if there exists a bisimulation $Z$ between $\mathfrak{M}$ and $\mathfrak{M}^{\prime}$ such that $w Z w^{\prime}$.

The notions of modal equivalence and bisimulation defined here follow very closely the standard classical notions; the only difference is that agreement of variables in bisimilar states and formulas in modally equivalent states means that they take the same values in those states. Moreover, the proof that bisimilarity implies modal equivalence is very similar to the classical proof (see, e.g., [3]).

Lemma 3 Let $\mathfrak{M}=\langle W, R, V\rangle$ and $\mathfrak{M}^{\prime}=\left\langle W^{\prime}, R^{\prime}, V^{\prime}\right\rangle$ be $\mathrm{K}(\mathbf{A})^{\mathrm{C}}$-models. If $w \in$ $W$ and $w^{\prime} \in W^{\prime}$ are bisimilar, then they are modally equivalent.

Proof We prove that for all $\varphi \in \mathrm{Fm}_{\square \diamond}, w \in W$, and $w^{\prime} \in W^{\prime}, w \equiv w^{\prime}$ implies $V(\varphi, w)=V^{\prime}\left(\varphi, w^{\prime}\right)$, proceeding by induction on $\ell(\varphi)$. For the case where $\varphi$ is a variable, the claim follows directly from the definition of a bisimulation. The cases of the propositional connectives follow immediately using the induction hypothesis. Consider then $\varphi=\diamond \psi$, the case $\varphi=\square \psi$ being very similar. Since $w \equiv w^{\prime}$, it follows by the forth condition that for each $v \in R[w]$, there exists $v^{\prime} \in R^{\prime}\left[w^{\prime}\right]$ such that $v \equiv v^{\prime}$ and, by the induction hypothesis, $V(\psi, v)=V^{\prime}\left(\psi, v^{\prime}\right)$. So $V(\diamond \varphi, w) \leq$ $V^{\prime}\left(\nabla \varphi, w^{\prime}\right)$. But also by the back condition, for each $v^{\prime} \in R^{\prime}\left[w^{\prime}\right]$, there exists $v \in$ $R[w]$ such that $v \equiv v^{\prime}$ and, by the induction hypothesis, $V(\psi, v)=V^{\prime}\left(\psi, v^{\prime}\right)$. So $V(\diamond \psi, w) \geq V^{\prime}\left(\diamond \psi, w^{\prime}\right)$. Hence $V(\diamond \psi, w)=V^{\prime}\left(\diamond \psi, w^{\prime}\right)$ as required.

Modal equivalence between states does not imply the bisimilarity of those states even in the classical case. Instead, we consider certain classes of models for which the two properties may or may not coincide, depending on the underlying chainbased algebra $\mathbf{A}$. We say that a class $\mathcal{K}$ of $\mathrm{K}(\mathbf{A})^{\mathrm{C}}$-models has the Hennessy-Milner property if for any models $\mathfrak{M}=\langle W, R, V\rangle$ and $\mathfrak{M}^{\prime}=\left\langle W^{\prime}, R^{\prime}, V^{\prime}\right\rangle$ in $\mathcal{K}$, whenever the states $w \in W$ and $w^{\prime} \in W^{\prime}$ are modally equivalent, they are also bisimilar. In the 
next section, we provide an algebraic characterization of chain-based algebras $\mathbf{A}$ for which the class of "image-finite" $\mathrm{K}(\mathbf{A})^{\mathrm{C}}$-models has the Hennessy-Milner property. In Section 4, we then adapt this characterization to a suitable notion of "modally saturated" $\mathrm{K}(\mathbf{A})^{\mathrm{C}}$-models. Finally, in Section 5, we use these characterizations to obtain precise descriptions for the case of BL-chains (divisible MTL-chains) that are finite or have lattice reduct $[0,1]$.

\section{Image-Finite Models}

Let us fix again a chain-based algebra $\mathbf{A}$. We call a $\mathrm{K}(\mathbf{A})^{\mathrm{C}}$-model image-finite if $R[w]$ is finite for each $w \in W$. The standard proof that the class of image-finite Kripke models for the classical modal logic $\mathrm{K}$ has the Hennessy-Milner property proceeds as follows (see [3]). Suppose, towards a contradiction, that there exist image-finite Kripke models $\mathfrak{M}=\langle W, R, V\rangle$ and $\mathfrak{M}^{\prime}=\left\langle W^{\prime}, R^{\prime}, V^{\prime}\right\rangle$ for which modal equivalence is not a bisimulation. Now assume (without loss of generality) that the forth condition fails. Then there are $w, v \in W$ and $w^{\prime} \in W^{\prime}$ such that $w \leadsto w^{\prime}$ and $R w v$, but for each $v_{i}^{\prime} \in R^{\prime}\left[w^{\prime}\right]=\left\{v_{1}^{\prime}, \ldots, v_{n}^{\prime}\right\}$, there is a formula $\varphi_{i}$ satisfying $V\left(\varphi_{i}, v\right)=0$ and $V^{\prime}\left(\varphi_{i}, v_{i}^{\prime}\right)=1$. Defining $\varphi=\square\left(\varphi_{1} \vee \cdots \vee \varphi_{n}\right)$, we obtain $V(\varphi, w)=0$ and $V^{\prime}\left(\varphi, w^{\prime}\right)=1$, contradicting $w \leadsto w^{\prime}$.

This reasoning extends easily to $\mathrm{K}(\mathbf{A})^{\mathrm{C}}$ in the case where all distinct pairs of values in $A$ can be distinguished by a one-variable propositional formula.

Lemma 4 Suppose that there exists $c \in A$ such that for any distinct $a, b \in A$, there exists a one-variable propositional formula $\psi_{a, b} \in \mathrm{Fm}$ satisfying $\psi_{a, b}[a] \geq c$ and $\psi_{a, b}[b]<c$. Then the class of image-finite $\mathrm{K}(\mathbf{A})^{\mathrm{C}}$-models has the Hennessy-Milner property.

Proof We revisit the proof for the classical case. Suppose for a contradiction that there are image-finite $\mathrm{K}(\mathbf{A})^{\mathrm{C}}$-models $\mathfrak{M}=\langle W, R, V\rangle$ and $\mathfrak{M}^{\prime}=\left\langle W^{\prime}, R^{\prime}, V^{\prime}\right\rangle$ for which modal equivalence is not a bisimulation. Assuming (without loss of generality) that the forth condition does not hold, there are $w, v \in W$ and $w^{\prime} \in W^{\prime}$ such that $w \leadsto w^{\prime}$ and $R w v$, but for each $v_{i}^{\prime} \in R^{\prime}\left[w^{\prime}\right]=\left\{v_{1}^{\prime}, \ldots, v_{n}^{\prime}\right\}$, there exists $\varphi_{i} \in$ Fm $\square \diamond$ satisfying $V\left(\varphi_{i}, v\right) \neq V^{\prime}\left(\varphi_{i}, v_{i}^{\prime}\right)$. Let $a_{i}=V^{\prime}\left(\varphi_{i}, v_{i}^{\prime}\right)$ and $b_{i}=V\left(\varphi_{i}, v\right)$ for each $i \in\{1, \ldots, n\}$. Then, by assumption, there is a one-variable propositional formula $\psi_{i}(p)$ for each $i \in\{1, \ldots, n\}$ such that $\psi_{i}\left[a_{i}\right] \geq c$ and $\psi_{i}\left[b_{i}\right]<c$. We define

$$
\varphi=\square\left(\psi_{1}\left[\varphi_{1} / p\right] \vee \cdots \vee \psi_{n}\left[\varphi_{n} / p\right]\right)
$$

Then, using Lemma 1 and the linearity of $\mathbf{A}$,

$$
\begin{aligned}
V(\varphi, w) & \leq V\left(\psi_{1}\left[\varphi_{1} / p\right], v\right) \vee \cdots \vee V\left(\psi_{n}\left[\varphi_{n} / p\right], v\right) \\
& =\psi_{1}\left[b_{1}\right] \vee \cdots \vee \psi_{n}\left[b_{n}\right] \\
& <c
\end{aligned}
$$


but also

$$
\begin{aligned}
V^{\prime}\left(\varphi, w^{\prime}\right) & =\bigwedge_{i=1}^{n} V^{\prime}\left(\psi_{1}\left[\varphi_{1} / p\right] \vee \cdots \vee \psi_{n}\left[\varphi_{n} / p\right], v_{i}^{\prime}\right) \\
& \geq \bigwedge_{i=1}^{n} V^{\prime}\left(\psi_{i}\left[\varphi_{i} / p\right], v_{i}^{\prime}\right) \\
& =\bigwedge_{i=1}^{n} \psi_{i}\left[a_{i}\right] \\
& \geq c .
\end{aligned}
$$

This contradicts $w \leftrightarrow w^{\prime}$.

Example 3 Consider the chain-based algebra for three-valued Łukasiewicz logic

$$
\mathrm{Ł}_{\mathbf{3}}=\left\langle\left\{0, \frac{1}{2}, 1\right\}, \min , \max , \cdot_{\mathrm{七}}, \rightarrow_{\mathrm{七}}, 0,1\right\rangle,
$$

where $x \cdot_{七} y=\max (0, x+y-1)$ and $x \rightarrow_{七} y=\min (1,1-x+y)$. We can distinguish values (with respect to 1 ) using the propositional formulas

$$
\begin{array}{lll}
\psi_{1,0}=(p \leftrightarrow \top), & \psi_{1, \frac{1}{2}}=(p \cdot p), & \psi_{\frac{1}{2}, 0}=(\neg p \rightarrow p), \\
\psi_{0,1}=(p \leftrightarrow \perp), & \psi_{\frac{1}{2}, 1}=(p \rightarrow \neg p), & \psi_{0, \frac{1}{2}}=(\neg p \cdot \neg p) .
\end{array}
$$

Hence, by Lemma 4, the class of image-finite $\mathrm{K}\left(\mathrm{七}_{3}\right)^{\mathrm{C}}$-models has the HennessyMilner property. Indeed, as we will see in Section 5, essentially the same reasoning can be used to show that the property holds for the class of image-finite models in the case of any finite-valued Łukasiewicz logic.

However, it is not the case that the class of image-finite $K(\mathbf{A})^{\mathrm{C}}$-models has the Hennessy-Milner property for any chain-based algebra A.

Example 4 Gödel logics are defined over chain-based algebras of the form

$$
\mathbf{A}=\left\langle A, \min , \max , \rightarrow_{\mathrm{G}}, 0,1\right\rangle,
$$

where $A$ is a complete subset of $[0,1]$ containing 0 and 1 , and $x \rightarrow_{\mathrm{G}} y=y$ if $x>y$ and $x \rightarrow_{\mathrm{G}} y=1$ otherwise. Suppose that $|A|>3$ and $0<a<b<c$ in A. Consider the $\mathrm{K}(\mathbf{A})^{\mathrm{C}}$-models $\mathfrak{M}=\langle W, R, V\rangle$ and $\mathfrak{M}^{\prime}=\left\langle W^{\prime}, R^{\prime}, V^{\prime}\right\rangle$ displayed in Fig. 1 with $W=\left\{w_{0}, w_{1}, w_{2}, w_{3}\right\}$ and $W^{\prime}=\left\{v_{0}, v_{1}, v_{2}\right\}, R$ and $R^{\prime}$ as indicated by the arrows, and $V$ and $V^{\prime}$ with the displayed values of $p$ and all other values 1 . Then it is easily shown (e.g., by considering the non-equivalent one-variable formulas) that $w_{0}$ and $v_{0}$ are modally equivalent. However, they are not bisimilar, as there is no state in $W^{\prime}$ corresponding to $w_{2}$. So the class of image-finite $\mathrm{K}(\mathbf{A})^{\mathrm{C}}$-models does not have the Hennessy-Milner property.

Example 5 Consider the chain-based algebra for product logic

$$
\mathbf{P}=\left\langle[0,1], \min , \max , \cdot \mathrm{P}, \rightarrow_{\mathrm{P}}, 0,1\right\rangle,
$$

where $x \cdot \mathrm{p} y=x y$ (multiplication) and $x \rightarrow_{\mathrm{p}} y$ is $\frac{y}{x}$ for $x>y$ and $x \rightarrow_{\mathrm{p}} y=1$ otherwise. Choose $c \in(0,1)$ and let $b=c^{2}$ and $a=c^{3}$, noting that $0<a<$ $b<c<1$. Consider again the models $\mathfrak{M}=\langle W, R, V\rangle$ and $\mathfrak{M}^{\prime}=\left\langle W^{\prime}, R^{\prime}, V^{\prime}\right\rangle$ displayed in Fig. 1 with $W=\left\{w_{0}, w_{1}, w_{2}, w_{3}\right\}$ and $W^{\prime}=\left\{v_{0}, v_{1}, v_{2}\right\}, R$ and $R^{\prime}$ as indicated by the arrows, and $V$ and $V^{\prime}$ with the displayed values of $p$ and all 

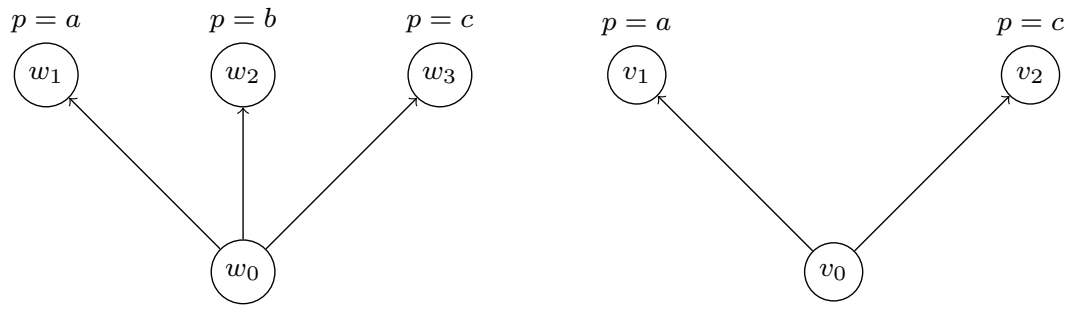

Fig. 1 Failure of the Hennessy-Milner property

other values 1 . Clearly, $w_{0}$ and $v_{0}$ are not bisimilar. To see that they are modally equivalent, consider any propositional formula $\psi(p) \in \mathrm{Fm}$. An easy induction on $\ell(\psi)$ establishes that $\psi$ restricted to $(0,1]$ is equivalent to $\perp$ or $p^{k}$ for some $k \in \mathbb{N}$. But then $V\left(\square \psi, w_{0}\right)=V^{\prime}\left(\square \psi, v_{0}\right)$ and $V\left(\diamond \psi, w_{0}\right)=V^{\prime}\left(\diamond \psi, v_{0}\right)$ for all $\psi \in \mathrm{Fm}$. By Lemma $2, w_{0}$ and $v_{0}$ are modally equivalent. So the class of $\mathrm{K}(\mathbf{P})^{\mathrm{C}}$-image-finite models does not have the Hennessy-Milner property.

Finding propositional formulas $\psi_{a, b}$, as described in Lemma 4, that distinguish distinct elements $a, b \in A$ is a sufficient condition for the class of image-finite $\mathrm{K}(\mathbf{A})^{\mathrm{C}}$-models to have the Hennessy-Milner property, but not a necessary one. Consider, for example, $A=\left\{0, \frac{1}{2}, 1\right\}$ in Example 4, giving an algebra $\mathbf{A}$ for three-valued Gödel logic. It is easy to show (by considering all possibilities) that there is no onevariable propositional formula $\psi$ in this case such that $\psi\left[\frac{1}{2}\right]>\psi[1]$, from which it follows that the lemma cannot be applied. On the other hand, the class of imagefinite $\mathrm{K}(\mathbf{A})^{\mathrm{C}}$-models does have the Hennessy-Milner property, as will be shown in Section 5. To obtain a complete characterization, we introduce a more complicated but still purely algebraic condition for $\mathbf{A}$.

Let $\mathbf{a} \in A^{n}$ and $\mathbf{C}=\left(\mathbf{c}_{1}, \ldots, \mathbf{c}_{n}\right) \in A^{n \times n}$. We call a propositional formula $\psi\left(p_{1}, \ldots, p_{n}\right) \in \mathrm{Fm}$ an $\mathbf{a} / \mathbf{C}$-distinguishing formula if

$$
\psi[\mathbf{a}]<\psi\left[\mathbf{c}_{i}\right] \text { for all } i \in\{1, \ldots, n\} \quad \text { or } \quad \psi[\mathbf{a}]>\psi\left[\mathbf{c}_{i}\right] \text { for all } i \in\{1, \ldots, n\} .
$$

We say that $\mathbf{A}$ has the distinguishing formula property if for all $n \in \mathbb{Z}^{+}, \mathbf{a} \in A^{n}$, and $\mathbf{C}=\left(\mathbf{c}_{1}, \ldots, \mathbf{c}_{n}\right) \in A^{n \times n}$ such that $\mathbf{a} \neq \mathbf{c}_{i}$ for all $i \in\{1, \ldots, n\}$, there is an a/C-distinguishing formula.

We establish the following characterization, noting that this theorem also holds (with an almost identical proof) for the box and diamond fragments of $\mathrm{K}(\mathbf{A})^{\mathrm{C}}$ if we restrict the distinguishing formula property to the first and second conditions, respectively.

Theorem 1 The class of image-finite $\mathrm{K}(\mathbf{A})^{\mathrm{C}}$-models has the Hennessy-Milner property if and only if $\mathbf{A}$ has the distinguishing formula property.

Proof Assume that $\mathbf{A}$ has the distinguishing formula property and suppose for a contradiction that there are two image-finite $\mathrm{K}(\mathbf{A})^{\mathrm{C}}$-models $\mathfrak{M}=\langle W, R, V\rangle$ and $\mathfrak{M}^{\prime}=\left\langle W^{\prime}, R^{\prime}, V^{\prime}\right\rangle$ for which modal equivalence is not a bisimulation. If $w \leadsto w^{\prime}$ 
for $w \in W$ and $w^{\prime} \in W^{\prime}$, then by definition $V(p, w)=V^{\prime}\left(p, w^{\prime}\right)$ for all $p \in$ Var, so the back condition or forth condition must be violated.

Let us suppose that the forth condition fails, the back condition being very similar. Then there exist $w, v \in W$ and $w^{\prime} \in W^{\prime}$ such that

1. $w \leadsto w^{\prime}$ and $R w v$.

2. No $v^{\prime} \in W^{\prime}$ satisfies both $R^{\prime} w^{\prime} v^{\prime}$ and $v \leadsto v^{\prime}$.

If $R^{\prime}\left[w^{\prime}\right]=\emptyset$, then consider $\diamond \top$. We have $V(\diamond \top, w)=\top$, but $V^{\prime}\left(\diamond \top, w^{\prime}\right)=\bigvee \emptyset=$ $\perp$, which contradicts $w \leadsto w^{\prime}$. Suppose then that $R^{\prime}\left[w^{\prime}\right]$ is non-empty and (because of image-finiteness) finite, say $R^{\prime}\left[w^{\prime}\right]=\left\{v_{1}^{\prime}, \ldots, v_{n}^{\prime}\right\}$. So there are formulas $\varphi_{i}$ such that $V\left(\varphi_{i}, v\right) \neq V^{\prime}\left(\varphi_{i}, v_{i}^{\prime}\right)$ for each $i \in\{1, \ldots, n\}$.

We define $\mathbf{a}=\left(a_{1}, \ldots, a_{n}\right)$ and $\mathbf{C}=\left(\mathbf{c}_{1}, \ldots, \mathbf{c}_{n}\right)$ with $\mathbf{c}_{i}=\left(c_{i, 1}, \ldots, c_{i, n}\right)$ by

$$
a_{i}=V\left(\varphi_{i}, v\right) \quad \text { and } \quad c_{i, j}=V^{\prime}\left(\varphi_{j}, v_{i}^{\prime}\right) \quad \text { for } i, j \in\{1, \ldots, n\} \text {. }
$$

Note that $\mathbf{a} \neq \mathbf{c}_{i}$ for each $i \in\{1, \ldots, n\}$ (because $a_{i} \neq c_{i, i}$ ).

By the distinguishing formula property, there exists an $\mathbf{a} / \mathbf{C}$-distinguishing propositional formula $\psi\left(p_{1}, \ldots, p_{n}\right) \in \mathrm{Fm}$. Suppose that

$$
\psi[\mathbf{a}]<\psi\left[\mathbf{c}_{i}\right] \text { for all } i \in\{1, \ldots, n\},
$$

the case where $\psi[\mathbf{a}]>\psi\left[\mathbf{c}_{i}\right]$ for all $i \in\{1, \ldots, n\}$ being very similar. Now define

$$
\varphi=\square \psi\left[\varphi_{1} / p_{1}, \ldots, \varphi_{n} / p_{n}\right] .
$$

Then using Lemma 1 and the linearity of $\mathbf{A}$,

$$
\begin{aligned}
V(\varphi, w) & \leq V\left(\psi\left[\varphi_{1} / p_{1}, \ldots, \varphi_{n} / p_{n}\right], v\right) \\
& =\psi\left[V\left(\varphi_{1}, v\right), \ldots, V\left(\varphi_{n}, v\right)\right] \\
& =\psi[\mathbf{a}] \\
& <\bigwedge_{i=1}^{n} \psi\left[\mathbf{c}_{i}\right] \\
& =\bigwedge_{i=1}^{n} \psi\left[V^{\prime}\left(\varphi_{1}, v_{i}^{\prime}\right), \ldots, V^{\prime}\left(\varphi_{n}, v_{i}^{\prime}\right)\right] \\
& =\bigwedge_{i=1}^{n} V^{\prime}\left(\psi\left[\varphi_{1} / p_{1}, \ldots, \varphi_{n} / p_{n}\right], v_{i}^{\prime}\right) \\
& =V^{\prime}\left(\varphi, w^{\prime}\right) .
\end{aligned}
$$

This contradicts $w \longleftrightarrow w^{\prime}$.

Suppose now conversely that the class of image-finite $\mathrm{K}(\mathbf{A})^{\mathrm{C}}$-models has the Hennessy-Milner property. For $n \in \mathbb{Z}^{+}, \mathbf{a} \in A^{n}$, and $\mathbf{C}=\left(\mathbf{c}_{1}, \ldots, \mathbf{c}_{n}\right) \in A^{n \times n}$ such that $\mathbf{a} \neq \mathbf{c}_{i}$ for all $i \in\{1, \ldots, n\}$, we seek an a/C-distinguishing propositional formula $\psi\left(p_{1}, \ldots, p_{n}\right) \in \mathrm{Fm}$. Consider two image-finite $\mathrm{K}(\mathbf{A})^{\mathrm{C}}$-models $\mathfrak{M}=\langle W, R, V\rangle$ and $\mathfrak{M}^{\prime}=\left\langle W^{\prime}, R^{\prime}, V^{\prime}\right\rangle$ such that

1. $W=\left\{w, v_{1}, \ldots, v_{n}, v\right\}$ and $W^{\prime}=\left\{w^{\prime}, v_{1}^{\prime}, \ldots, v_{n}^{\prime}\right\}$

2. $R=\left\{\left(w, v_{i}\right) \mid 1 \leq i \leq n\right\} \cup\{(w, v)\}$ and $R^{\prime}=\left\{\left(w^{\prime}, v_{i}^{\prime}\right) \mid 1 \leq i \leq n\right\}$

3. $V\left(p_{j}, v_{i}\right)=V^{\prime}\left(p_{j}, v_{i}^{\prime}\right)=c_{i, j}$ and $V\left(p_{j}, v\right)=a_{j}$ for $1 \leq i, j \leq n$, and $V, V^{\prime}$ are constantly $\top$ elsewhere. 
Observe that $w$ and $w^{\prime}$ are not bisimilar; there is no state in $W^{\prime}$ accessible from $w^{\prime}$ that agrees with $v$ on all propositional variables since $\mathbf{a} \neq \mathbf{c}_{i}$ for each $i \in\{1, \ldots, n\}$.

Hence, by the Hennessy-Milner property for image-finite $\mathrm{K}(\mathbf{A})^{\mathrm{C}}$-models, $w$ and $w^{\prime}$ are not modally equivalent. Using Lemma 2, it follows that $V(\varphi, w) \neq V^{\prime}\left(\varphi, w^{\prime}\right)$ for some formula $\varphi=\square \psi$ or $\varphi=\diamond \psi$ where $\psi \in$ Fm contains only the variables $p_{1}, \ldots, p_{n}$. Suppose that $\varphi=\square \psi$, the case $\varphi=\diamond \psi$ being very similar. Clearly $V(\square \psi, w) \leq V^{\prime}\left(\square \psi, w^{\prime}\right)$, so for each $i \in\{1, \ldots, n\}$,

$$
\psi[\mathbf{a}]=V(\psi, v)=V(\square \psi, w)<V^{\prime}\left(\square \psi, w^{\prime}\right) \leq V^{\prime}\left(\psi, v_{i}^{\prime}\right)=\psi\left[\mathbf{c}_{i}\right] .
$$

That is, $\psi$ is the required a/C-distinguishing formula.

\section{Modally Saturated Models}

We first recall the definition of modally saturated models for classical modal logic. Consider a (classical) Kripke model $\mathfrak{M}=\langle W, R, V\rangle$. A set $\Sigma$ of (classical modal) formulas is satisfiable in $X \subseteq W$ if there exists $w \in X$ satisfying $V(\varphi, w)=1$ for all $\varphi \in \Sigma$; it is finitely satisfiable in $X$ if every finite subset of $\Sigma$ is satisfiable in $X$. The model $\mathfrak{M}$ is (classically) modally saturated if for every $w \in W$, any set $\Sigma$ of formulas that is finitely satisfiable in $R[w]$ is also satisfiable in $R[w]$.

We now generalize this notion to the many-valued setting, fixing again a chainbased algebra $\mathbf{A}$. Instead of satisfaction of a set of formulas at some state, we specify the exact values that formulas should take at some state. Intuitively, this is achieved by assigning values from $\mathbf{A}$ to formulas in such a way that the propositional operations are preserved. More formally, let $\mathbf{F m}^{*}$ be the formula algebra of $\mathcal{L}$ over the set of variables $\operatorname{Var} \cup\left\{\square \varphi, \diamond \varphi \mid \varphi \in \mathrm{Fm}_{\square \diamond}\right\}$, noting that $\mathrm{Fm}^{*}=\mathrm{Fm}_{\square \diamond}$. We call a homomorphism $U: \mathbf{F m}^{*} \rightarrow \mathbf{A}$ a pseudo-valuation for $\mathbf{A}$. Note in particular that for any $\mathrm{K}(\mathbf{A})^{\mathrm{C}}$-model $\mathfrak{M}=\langle W, R, V\rangle$ and $w \in W$, the map

$$
V_{w}: \mathrm{Fm}_{\square \diamond} \rightarrow \mathbf{A} ; \quad \varphi \mapsto \mathrm{V}(\varphi, \mathrm{w})
$$

is a pseudo-valuation. Indeed, such pseudo-valuations will play a key role in the proof of the main theorem below.

Now consider a $\mathrm{K}(\mathbf{A})^{\mathrm{C}}$-model $\mathfrak{M}=\langle W, R, V\rangle$ and a pseudo-valuation $U$ for A. We say that $\Sigma \subseteq \mathrm{Fm}_{\square \diamond}$ is $U$-satisfiable in $X \subseteq W$ if there exists $w \in X$ satisfying $V(\varphi, w)=U(\varphi)$ for all $\varphi \in \Sigma$; it is finitely $U$-satisfiable in $X$ if every finite subset of $\Sigma$ is $U$-satisfiable in $X$. We call $\mathfrak{M}$ modally saturated if for every $w \in W$ and pseudo-valuation $U$ for $\mathbf{A}$, any subformula-closed $\Sigma \subseteq \mathrm{Fm}_{\square \diamond}$ that is finitely $U$-satisfiable in $R[w]$ is also $U$-satisfiable in $R[w]$.

In the case where $\mathbf{A}$ is the two-valued Boolean algebra 2 , this notion coincides with the usual classical definition. Suppose that a $\mathrm{K}(\mathbf{2})^{\mathrm{C}}$-model $\mathfrak{M}=\langle W, R, V\rangle$ is classically modally saturated and consider $w \in W$, a pseudo-valuation $U$ for 2 , and a subformula-closed $\Sigma \subseteq \mathrm{Fm}_{\square \diamond}$ that is finitely $U$-satisfiable in $R[w]$. Let

$$
\Gamma=\{\varphi \mid \varphi \in \Sigma \text { and } U(\varphi)=1\} \cup\{\neg \varphi \mid \varphi \in \Sigma \text { and } U(\varphi)=0\} .
$$

Then $\Gamma$ is finitely satisfiable in $R[w]$ and hence, by assumption, satisfiable in $R[w]$. By the definition of $\Gamma$, it follows that $\Sigma$ is also $U$-satisfiable in $R[w]$. 
Suppose now conversely that $\mathfrak{M}$ is modally saturated and consider $w \in W$ and a set $\Sigma \subseteq \mathrm{Fm}_{\square \diamond}$ that is finitely satisfiable in $R[w]$. Observe that for any $\varphi \in \mathrm{Fm}_{\square \diamond}$, either $\Sigma \cup\{\varphi\}$ or $\Sigma \cup\{\neg \varphi\}$ is finitely satisfiable in $R[w]$. Hence $\Sigma$ extends to a set $\Sigma^{*} \subseteq \mathrm{Fm}_{\square \diamond}$ that is maximally finitely satisfiable in $R[w]$. Define

$$
U(\varphi)= \begin{cases}1 & \text { if } \varphi \in \Sigma^{*} \\ 0 & \text { otherwise }\end{cases}
$$

Observe that $U\left(\varphi_{1} \wedge \varphi_{2}\right)=1$ if and only if $U\left(\varphi_{1}\right)=U\left(\varphi_{2}\right)=1$ and $U(\neg \varphi)=1$ if and only if $U(\varphi)=0$, so $U$ is a pseudo-valuation for 2 . Now consider any finite subset of $F m_{\square \diamond}$, which we can assume to be of the form $\Sigma_{1} \cup \Sigma_{2}$, where $\Sigma_{1} \cup\{\neg \varphi \mid$ $\left.\varphi \in \Sigma_{2}\right\} \subseteq \Sigma^{*}$. Since $\Sigma^{*}$ is finitely satisfiable in $R[w], \Sigma_{1} \cup\left\{\neg \varphi \mid \varphi \in \Sigma_{2}\right\}$ is satisfiable in $R[w]$. But then also $\Sigma_{1} \cup \Sigma_{2}$ is $U$-satisfiable in $R[w]$. So Fm $\square \diamond$ is finitely $U$-satisfiable in $R[w]$ and hence, by assumption, $U$-satisfiable in $R[w]$. In particular, $\Sigma$ is satisfiable in $R[w]$.

We may also confirm that $\mathrm{m}$-saturation is a generalization of image-finiteness.

Lemma 5 Every image-finite $\mathrm{K}(\mathbf{A})^{\mathrm{C}}$-model is modally saturated.

Proof Let $\mathfrak{M}=\langle W, R, V\rangle$ be an image-finite $\mathrm{K}(\mathbf{A})^{\mathrm{C}}$-model, $w \in W$, and $U$ a pseudo-valuation for A. Suppose contrapositively that a subformula-closed $\Gamma \subseteq$ $\mathrm{Fm}_{\square \diamond}$ is not $U$-satisfiable in $R[w]$. Since $\mathfrak{M}$ is image-finite, $R[w]$ is finite, say $R[w]=\left\{v_{1}, \ldots, v_{n}\right\}$. So for each $i \in\{1, \ldots, n\}$, there is a formula $\varphi_{i} \in \Gamma$ such that $V\left(\varphi_{i}, v_{i}\right) \neq U\left(\varphi_{i}\right)$. But then also the finite set $\left\{\varphi_{1}, \ldots, \varphi_{n}\right\} \subseteq \Gamma$ is not $U$-satisfiable in $R[w]$.

The converse to this lemma does not hold. Consider the $\mathrm{K}(\mathbf{A})^{\mathrm{C}}$-model $\mathfrak{M}=$ $\langle W, R, V\rangle$ with $W=\mathbb{N}, R=\{(0, n) \mid n \in \mathbb{N}\}$, and a constant valuation defined for some fixed $a \in A$ by $V(p, n)=a$ for all $p \in \operatorname{Var}$ and $n \in \mathbb{N}$. Obviously, this model is modally saturated, but not image-finite.

We note the following useful property.

Lemma 6 Let $\mathfrak{M}$ be a $\mathrm{K}(\mathbf{A})^{C}$-tree-model with root $w$ and height 1 such that $V$ is the constant function $\top$ on $(\operatorname{Var} \backslash P) \times W$ for some finite $P \subseteq$ Var. Then $\mathfrak{M}$ is modally saturated.

Proof Let $\mathfrak{M}$ be a $\mathrm{K}(\mathbf{A})^{\mathrm{C}}$-tree-model with root $w$ and height 1 such that $V$ is the constant function $\top$ on $(\operatorname{Var} \backslash P) \times W$ for some finite $P \subseteq$ Var. Consider a subformulaclosed set $\Sigma \subseteq \mathrm{Fm}_{\square \diamond}$. Let $Q$ be the set of variables occurring in both $P$ and $\Sigma$ and define the propositional set of formulas $\Sigma^{*}=\left\{\varphi^{*} \mid \varphi \in \Sigma\right\}$ where $\varphi^{*}$ is obtained from $\varphi$ by replacing every variable not in $Q$ by $\top$, every subformula $\square \psi$ by $\top$, and every subformula $\diamond \psi$ by $\perp$. A simple induction yields that $V(\varphi, v)=V\left(\varphi^{*}, v\right)$ for any $v \in R[w]$ and $\varphi \in \Sigma$. Now consider a pseudo-valuation $U$ for $\mathbf{A}$ such that $\Sigma$ is finitely $U$-satisfiable in $R[w]$. Clearly then also $\Sigma^{*}$ is finitely $U$-satisfiable in $R[w]$. In particular, since $\Sigma$ is subformula-closed, $Q \subseteq \Sigma$ and there exists $v \in R[w]$ such that $V(p, v)=U(p)$ for all $p \in Q$. Hence also $V(\varphi, v)=U\left(\varphi^{*}\right)$ for all $\varphi \in \Sigma$. Moreover, a simple induction on $\ell(\varphi)$ shows that $U\left(\varphi^{*}\right)=U(\varphi)$ for all $\varphi \in \Sigma$. So $\Sigma$ is $U$-satisfiable in $R[w]$. 
We now consider a stronger version of the distinguishing formula property which will allow us to give a characterization of the Hennessy-Milner property for classes of modally saturated models. Given a $\in A^{n}$, we say that a propositional formula $\psi\left(p_{1}, \ldots, p_{n}\right) \in \mathrm{Fm}$ is a strong distinguishing formula for $\mathbf{a}$ if

$$
\psi[\mathbf{a}]>\sup \left\{\psi[\mathbf{c}] \mid \mathbf{c} \in A^{n} \backslash\{\mathbf{a}\}\right\} \quad \text { or } \quad \psi[\mathbf{a}]<\inf \left\{\psi[\mathbf{c}] \mid \mathbf{c} \in A^{n} \backslash\{\mathbf{a}\}\right\} .
$$

We say that $\mathbf{A}$ has the strong distinguishing formula property if for all $n \in \mathbb{Z}^{+}$ and $\mathbf{a} \in A^{n}$, there is a strong distinguishing formula for $\mathbf{a}$. Note that if $\mathbf{A}$ has the strong distinguishing formula property, then it clearly has the distinguishing formula property. Moreover, if $\mathbf{A}$ is finite, then these two notions coincide.

We obtain the following characterization of the Hennessy-Milner property for classes of modally saturated models.

Theorem 2 The class of modally saturated $\mathrm{K}(\mathbf{A})^{\mathrm{C}}$-models has the Hennessy-Milner property if and only if $\mathbf{A}$ has the strong distinguishing formula property.

Proof Suppose that $\mathbf{A}$ has the strong distinguishing formula property and consider modally saturated $\mathrm{K}(\mathbf{A})^{\mathrm{C}}$-models $\mathfrak{M}=\langle W, R, V\rangle, \mathfrak{M}^{\prime}=\left\langle W^{\prime}, R^{\prime}, V^{\prime}\right\rangle$, and $w \in W$, $w^{\prime} \in W^{\prime}$ such that $w \leadsto w^{\prime}$. Assume for a contradiction that modal equivalence is not a bisimulation between $w$ and $w^{\prime}$. By definition, $w$ and $w^{\prime}$ agree on all propositional variables, so the forth or the back condition must be violated. Assume that the forth condition is violated, i.e., there is a $v \in R[w]$ but no $v^{\prime} \in R^{\prime}\left[w^{\prime}\right]$ such that $v$ $v^{\prime}$. Then $\mathrm{Fm}_{\square \diamond}$ is not $V_{v}$-satisfiable in $R^{\prime}\left[w^{\prime}\right]$. So, since $\mathfrak{M}^{\prime}$ is modally saturated, $\mathrm{Fm}_{\square \diamond}$ is not finitely $V_{v}$-satisfiable in $R^{\prime}\left[w^{\prime}\right]$. Hence there exists $\left\{\varphi_{1}, \ldots, \varphi_{n}\right\} \subseteq$ Fm $\square \diamond$ such that $\left\{\varphi_{1}, \ldots, \varphi_{n}\right\}$ is not $V_{v}$-satisfied in any $v^{\prime} \in R^{\prime}\left[w^{\prime}\right]$. We set

$$
\mathbf{a}=\left(V\left(\varphi_{1}, v\right), \ldots, V\left(\varphi_{n}, v\right)\right) .
$$

By the strong distinguishing formula property, there exists a propositional formula $\psi\left(p_{1}, \ldots, p_{n}\right) \in \mathrm{Fm}$ such that

$$
\psi[\mathbf{a}]>\sup \left\{\psi[\mathbf{c}] \mid \mathbf{c} \in A^{n} \backslash\{\mathbf{a}\}\right\} \quad \text { or } \quad \psi[\mathbf{a}]<\inf \left\{\psi[\mathbf{c}] \mid \mathbf{c} \in A^{n} \backslash\{\mathbf{a}\}\right\} .
$$

Suppose that $\psi[\mathbf{a}]>\sup \left\{\psi[\mathbf{c}] \mid \mathbf{c} \in A^{n} \backslash\{\mathbf{a}\}\right\}$, the other case being very similar. For each $v^{\prime} \in R^{\prime}\left[w^{\prime}\right]$, let $\mathbf{c}_{v^{\prime}}=\left(V^{\prime}\left(\varphi_{1}, v^{\prime}\right), \ldots,\left(V^{\prime}\left(\varphi_{n}, v^{\prime}\right)\right)\right.$, observing that $\mathbf{a} \neq \mathbf{c}_{v^{\prime}}$, and define the modal formula

$$
\tilde{\psi}=\psi\left[\varphi_{1} / p_{1}, \ldots, \varphi_{n} / p_{n}\right] .
$$

Using Lemma 1, we obtain

$$
\begin{aligned}
V(\diamond \tilde{\psi}, w) & \geq V(\tilde{\psi}, v) \\
& =\psi[\mathbf{a}] \\
& >\sup \left\{\psi\left[\mathbf{c}_{v^{\prime}}\right] \mid v^{\prime} \in R^{\prime}\left[w^{\prime}\right]\right\} \\
& =\sup \left\{V^{\prime}\left(\tilde{\psi}, v^{\prime}\right) \mid v^{\prime} \in R^{\prime}\left[w^{\prime}\right]\right\} \\
& =V^{\prime}\left(\diamond \tilde{\psi}, w^{\prime}\right),
\end{aligned}
$$

contradicting $w \leadsto w^{\prime}$. The case where the back condition fails is analogous. 
Now assume conversely that the class of modally saturated $\mathrm{K}(\mathbf{A})^{\mathrm{C}}$-models has the Hennessy-Milner property. Consider $\mathbf{a} \in A^{n}$ and variables $\left\{p_{1}, \ldots, p_{n}\right\} \subseteq$ Var. We define $\mathrm{K}(\mathbf{A})^{\mathrm{C}}$-models $\mathfrak{M}=\langle W, R, V\rangle$ and $\mathfrak{M}^{\prime}=\left\langle W^{\prime}, R^{\prime}, V^{\prime}\right\rangle$ satisfying

1. $W=\left\{w, v_{\mathbf{a}}\right\} \cup\left\{v_{\mathbf{c}} \mid \mathbf{c} \in A^{n} \backslash\{\mathbf{a}\}\right\}$ and $W^{\prime}=\left\{w^{\prime}\right\} \cup\left\{v_{\mathbf{c}}^{\prime} \mid \mathbf{c} \in A^{n} \backslash\{\mathbf{a}\}\right\}$

2. $R=\left\{\left(w, v_{\mathbf{a}}\right)\right\} \cup\left\{\left(w, v_{\mathbf{c}}\right) \mid \mathbf{c} \in A^{n} \backslash\{\mathbf{a}\}\right\}$ and $R^{\prime}=\left\{\left(w^{\prime}, v_{\mathbf{c}}^{\prime}\right) \mid \mathbf{c} \in A^{n} \backslash\{\mathbf{a}\}\right\}$

3. $V\left(p_{i}, v_{\mathbf{a}}\right)=a_{i}$ and $V\left(p_{i}, v_{\mathbf{c}}\right)=V^{\prime}\left(p_{i}, v_{\mathbf{c}}^{\prime}\right)=c_{i}$ for $1 \leq i \leq n$ and $\mathbf{c} \in A^{n} \backslash\{\mathbf{a}\}$, and $V, V^{\prime}$ are constantly $\top$ elsewhere.

It follows from Lemma 6 that both $\mathfrak{M}$ and $\mathfrak{M}^{\prime}$ are modally saturated. Clearly, $w$ and $w^{\prime}$ are not bisimilar, since there is no state in $R^{\prime}\left[w^{\prime}\right]$ corresponding to $v_{\mathbf{a}}$. By the Hennessy-Milner property, $w$ and $w^{\prime}$ are not modally equivalent, so there exists a formula $\varphi$ such that $V(\varphi, w) \neq V^{\prime}\left(\varphi, w^{\prime}\right)$. By Lemma 2, it follows that $V(\varphi, w) \neq$ $V^{\prime}\left(\varphi, w^{\prime}\right)$ for some formula $\varphi=\square \psi$ or $\varphi=\diamond \psi$ where $\psi \in \mathrm{Fm}$ is a propositional formula. Suppose that $\varphi=\diamond \psi$, the other case being very similar. Then $V(\diamond \psi, w)>$ $V^{\prime}\left(\diamond \psi, w^{\prime}\right)$ and

$$
\begin{aligned}
\psi[\mathbf{a}] & =V\left(\psi, v_{\mathbf{a}}\right) \\
& >\sup \left\{V^{\prime}\left(\psi, v_{\mathbf{c}}^{\prime}\right) \mid \mathbf{c} \in A^{n} \backslash\{\mathbf{a}\}\right\} \\
& =\sup \left\{\psi[\mathbf{c}] \mid \mathbf{c} \in A^{n} \backslash\{\mathbf{a}\}\right\} .
\end{aligned}
$$

That is, $\mathbf{A}$ has the strong distinguishing formula property.

\section{Divisible Chain-Based Modal Logics}

In this section, we provide full characterizations for finite and standard BL-chains (see Example 1), describing exactly when the classes of image-finite and modally saturated $\mathrm{K}(\mathbf{A})^{\mathrm{C}}$-models of such an algebra $\mathbf{A}$ admit the Hennessy-Milner property. For convenience, we exploit the fact that $a \wedge b=a \cdot(a \rightarrow b)$ and $a \vee b=((a \rightarrow$ $b) \rightarrow b) \wedge((b \rightarrow a) \rightarrow a)$ for all $a, b \in A$, and restrict to the (usual) language of BL-algebras with operation symbols $\cdot, \rightarrow, \perp, \top$.

We consider first the special case where $\mathbf{A}$ is an $M V$-chain, defined (up to term equivalence) as a BL-chain satisfying the involution property $\neg \neg a=a$ for all $a \in A$. Consider in particular the MV-chains

$$
\begin{aligned}
& \mathbf{L}_{\mathbf{n}+\mathbf{1}}=\left\langle\left\{0, \frac{1}{n}, \ldots, \frac{n-1}{n}, 1\right\},{ }_{\mathfrak{七}}, \rightarrow_{\mathfrak{七}}, 0,1\right\rangle \quad\left(n \in \mathbb{Z}^{+}\right) \\
& \mathbf{t}_{\infty}=\left\langle[0,1], \cdot_{\mathfrak{E}}, \rightarrow_{€}, 0,1\right\rangle,
\end{aligned}
$$

where $x \cdot_{七} y=\max (0, x+y-1)$ and $x \rightarrow_{€} y=\min (1,1-x+y)$. For convenience, fix also $\mathbf{L}_{1}$ to be an MV-chain with one element. Then every finite $M V$-chain $\mathbf{A}$ is isomorphic to $\mathbf{L}_{|A|}$ and every standard MV-chain is isomorphic to $\mathbf{L}_{\infty}$ (see [9] for proofs and a wealth of further details for MV-algebras).

We use McNaughton's description of the free one-generated MV-algebra to distinguish between rational values in $[0,1]$. A unary McNaughton function is a continuous function $f:[0,1] \rightarrow[0,1]$ with the property that there exist linear functions $g_{1}, \ldots, g_{k}$ with integer coefficients such that for any $x \in[0,1]$, there is an $i \in\{1, \ldots, k\}$ satisfying $f(x)=g_{i}(x)$. 
Theorem 3 (McNaughton [22]) The free one-generated MV-algebra is isomorphic to the algebra of unary McNaughton functions equipped with pointwise defined operations.

Lemma 7 Let $\mathbf{A}$ be an $M V$-chain.

(a) If $\mathbf{A}$ is finite, then the classes of image-finite and modally saturated $\mathrm{K}(\mathbf{A})^{\mathrm{C}}$ models have the Hennessy-Milner property.

(b) If $\mathbf{A}$ is standard, then the class of image-finite $\mathrm{K}(\mathbf{A})^{\mathrm{C}}$-models has the HennessyMilner property, but not the class of modally saturated $\mathrm{K}(\mathbf{A})^{\mathrm{C}}$-models.

Proof By the above remarks, we need only consider the case where $\mathbf{A}$ is $\mathbf{L}_{\alpha}$ for $\alpha \in \mathbb{Z}^{+} \cup\{\infty\}$. Consider distinct $a, b \in A$. Suppose that $a<b$, the case $a>b$ being very similar. Then there exist $c, d \in \mathbb{Q}$ such that $a<c<d<b$ and we can define $f$ to be 1 on the interval $[0, c], 0$ on the interval $[d, 1]$, and linear on $(c, d)$. Using Theorem 3, there exists a propositional formula $\psi_{a, b}(p)$ such that in the algebra $\mathbf{L}_{\infty}$, we have $\psi_{a, b}[x]=f(x)$ for all $x \in[0,1]$. Since $\mathbf{A}$ is a subalgebra of $\mathbf{L}_{\infty}$, also $\psi_{a, b}[a]=1$ and $\psi_{a, b}[b] \neq 1$. Hence, by Lemma 4 , the class of image-finite $\mathrm{K}(\mathbf{A})^{\mathrm{C}_{-}}$ models has the Hennessy-Milner property. If $\alpha \in \mathbb{Z}^{+}$, then $\mathbf{A}$ is finite and the same holds for the class of modally saturated $\mathrm{K}(\mathbf{A})^{\mathrm{C}}$-models. Finally, to show that the class of modally saturated $\mathrm{K}\left(\mathbf{L}_{\infty}\right)^{\mathrm{C}}$-models does not have the Hennessy-Milner property, observe that for any propositional formula $\psi[x]$, by continuity,

$$
\psi[1]=\sup \{\psi[c] \mid 0 \leq c<1\}=\inf \{\psi[c] \mid 0 \leq c<1\}
$$

So $\psi$ is not a strong distinguishing formula for 1 .

In order to extend this characterization to finite and standard BL-chains, we recall a useful description of the structure of these algebras. A hoop is an algebra $\mathbf{A}=$ $\langle A, \cdot, \rightarrow, \top\rangle$ such that $\langle A, \cdot, \top\rangle$ is a commutative monoid and for $a, b, c \in A$,

1. $a \rightarrow a=\top$

2. $a \cdot(a \rightarrow b)=b \cdot(b \rightarrow a)$

3. $a \rightarrow(b \rightarrow c)=(a \cdot b) \rightarrow c$.

Defining $a \leq b$ if and only if $a \rightarrow b=\top$ provides a semilattice order with meet operation $a \wedge b=a \cdot(a \rightarrow b)$ such that $\cdot$ and $\rightarrow$ are a residuated pair; i.e., $a \leq b \rightarrow c$ if and only if $a \cdot b \leq c$. If the order is linear, then $\mathbf{A}$ is called an o-hoop; if $A=[0,1]$ and $\leq$ is the usual order, then the o-hoop is called standard.

Suppose now that $\mathbf{A}_{i}=\left\langle A_{i}, \cdot_{i}, \rightarrow_{i}, \top\right\rangle$ is a non-trivial o-hoop for each $i$ in a linearly ordered set $I$ with bottom element $i_{0}$, where $A_{i} \cap A_{j}=\{\top\}$ for distinct $i, j \in I$ and $\mathbf{A}_{i_{0}}$ has a bottom element $\perp$. Then the ordinal sum of $\left(\mathbf{A}_{i}\right)_{i \in I}$ is

$$
\bigoplus_{i \in I} \mathbf{A}_{i}:=\left\langle\bigcup_{i \in I} A_{i}, \cdot, \rightarrow, \perp, \top\right\rangle
$$


where

$$
\begin{gathered}
x \cdot y= \begin{cases}x \cdot_{i} y & \text { if } x, y \in A_{i} \\
x & \text { if } x \in A_{i} \backslash\{\top\}, y \in A_{j}, \text { and } i<j \\
y & \text { if } y \in A_{i} \backslash\{\top\}, x \in A_{j}, \text { and } i<j\end{cases} \\
x \rightarrow y= \begin{cases}\top & \text { if } x \in A_{i} \backslash\{\top\}, y \in A_{j}, \text { and } i<j \\
x \rightarrow_{i} y & \text { if } x, y \in A_{i} \\
y & \text { if } y \in A_{i}, x \in A_{j}, \text { and } i<j .\end{cases}
\end{gathered}
$$

We also write $\mathbf{A}_{1} \oplus \cdots \oplus \mathbf{A}_{n}$ when $I=\{1, \ldots, n\}$ has the usual order.

Any ordinal sum of o-hoops is a BL-chain. Moreover, each "irreducible BLchain" A (a BL-chain that cannot be expressed as proper ordinal sums of o-hoops) is either (i) the hoop reduct $\langle A, \cdot, \rightarrow, \top\rangle$ of an MV-chain $\langle A, \cdot, \rightarrow, \perp, \top\rangle$, or (ii) a cancellative o-hoop, i.e., $a \rightarrow(a \cdot b)=b$ for all $a, b \in A$. Note that there are no finite cancellative o-hoops and that every standard cancellative o-hoop is isomorphic to the o-hoop

$$
\mathbf{C}=\left\langle(0,1], \cdot \mathrm{c}, \rightarrow_{\mathrm{C}}, 1\right\rangle
$$

where $x \cdot{ }^{\mathrm{C}} y=x y$ (multiplication) and $x \rightarrow_{\mathrm{C}} y$ is $\frac{y}{x}$ for $x>y$ and $x \rightarrow_{\mathrm{C}} y=1$ otherwise.

Theorem 4 (Aglianò and Montagna [1]) Every non-trivial BL-chain is the unique ordinal sum of a family of o-hoops each of which is either the hoop reduct of an $M V$-chain or a cancellative o-hoop.

The next two lemmas identify ordinal sums $\mathbf{A}$ of o-hoops such that the class of image-finite $\mathrm{K}(\mathbf{A})^{\mathrm{C}}$-models does or does not have the Hennessy-Milner property. For convenience, we let $\mathbf{A}^{\mathbf{h}}$ denote the hoop reduct of a BL-chain $\mathbf{A}$.

Lemma 8 Suppose that $\mathbf{A}$ is the ordinal sum of a family of (non-trivial) o-hoops $\left(\mathbf{A}_{i}\right)_{i \in I}$. If $|I| \geq 3$ or $\mathbf{A}_{i}$ is cancellative for some $i \in I$, then the class of image-finite $\mathrm{K}(\mathbf{A})^{\mathrm{C}}$-models does not have the Hennessy-Milner property.

Proof Suppose first that $\mathbf{A}_{i}$ is cancellative for some $i \in I$. Let $c \in A_{i} \backslash\{\top\}$ and define $b=c \cdot c$ and $a=c \cdot c \cdot c$, noting that, by cancellativity, $a<b<c<\top$. The failure of the Hennessy-Milner property then follows as described in Example 5 for product modal logic, considering $\mathbf{A}_{i}$ instead of $(0,1]$. Now consider the case where $|I| \geq 3$ and no $\mathbf{A}_{i}$ is cancellative for $i \in I$. Then, by Theorem 4, each $\mathbf{A}_{i}$ is a hoop reduct of an $\mathrm{MV}$-chain and has a bottom element distinct from the top element of $\mathbf{A}$ for $i \in I$. But these bottom elements and also the top element are idempotents (i.e., $a \cdot a=a$ ) and hence A contains as a subalgebra, a Gödel algebra with more than three elements. The failure of the Hennessy-Milner property then follows exactly as described in Example 4.

Lemma 9 Let $\mathbf{A}=\mathbf{L}_{\alpha}^{\mathbf{h}} \oplus \mathbf{L}_{\beta}^{\mathbf{h}}$ for some $\alpha, \beta \in \mathbb{Z}^{+} \cup\{\infty\}$. Then the class of imagefinite $\mathrm{K}(\mathbf{A})^{\mathrm{C}}$-models has the Hennessy-Milner property. 
Proof By Theorem 1, it suffices to show that $\mathbf{A}$ has the distinguishing formula property. Let $n \in \mathbb{Z}^{+}, \mathbf{a}=\left(a_{1}, \ldots, a_{n}\right) \in\left(\mathbf{七}_{\alpha}^{h} \oplus \mathbf{Ł}_{\beta}^{h}\right)^{n}$, and $\mathbf{C}=\left(\mathbf{c}_{1}, \ldots, \mathbf{c}_{n}\right) \in A^{n \times n}$ with $\mathbf{c}_{i} \neq \mathbf{a}$ for each $i \in\{1, \ldots, n\}$. We denote the bottom elements of $\succeq_{\alpha}$ and $\iota_{\beta}$ by $0_{1}$ and $0_{2}$, respectively. As a preliminary step, note that there exists $r \in \mathbb{Z}^{+}$such that for any element $b \neq 1$ occurring in a or $\mathbf{C}$, either $b \in \mathfrak{七}_{\alpha}^{h}$ and $b^{r}=0_{1}$ or $b \in \mathfrak{七}_{\beta}^{h}$ and $b^{r}=0_{2}$.

Suppose first that $\mathbf{a}=\left(a_{1}, \ldots, a_{m}, a_{m+1}, \ldots, a_{n}\right)$ (allowing for some rearrangement of the elements in a) where

$$
a_{1}, \ldots, a_{m} \in \mathrm{七}_{\alpha}^{h} \backslash\{1\} \quad \text { and } \quad a_{m+1}=\cdots=a_{n}=1 .
$$

We define a formula $\psi=\psi_{1} \wedge \psi_{2} \wedge \psi_{3}$ such that

$$
\psi[\mathbf{a}]=1 \quad \text { and } \quad \psi\left[\mathbf{c}_{i}\right]<1 \text { for all } i \in\{1, \ldots, n\} .
$$

1. Let $\psi_{1}=\neg p_{1}^{r} \wedge \cdots \wedge \neg p_{m}^{r}$ and observe that

$$
\psi_{1}\left[a_{1}, \ldots, a_{m}\right]=1 \text { and } \psi_{1}[\ldots, b, \ldots]=0_{1}<1 \text { for } b \in €_{\beta}^{h} .
$$

2. Using Theorem 3, for each $j \in\{1, \ldots, m\}$, there exists a propositional formula $\varphi_{j}\left(p_{j}\right)$ such that for all $i \in\{1, \ldots, n\}$,

$$
\varphi_{j}\left[a_{j}\right]=1 \quad \text { and } \quad \varphi_{j}\left[c_{i, j}\right]=0_{1} \text { if } c_{i, j} \in \mathrm{七}_{\alpha}^{h} \backslash\left\{a_{j}\right\} .
$$

Let $\psi_{2}=\varphi_{1} \wedge \cdots \wedge \varphi_{m}$ and observe that for all $i \in\{1, \ldots, n\}$,

$$
\psi_{2}\left[a_{1}, \ldots, a_{m}\right]=1 \quad \text { and } \quad \psi_{2}\left[\ldots, c_{i, j}, \ldots\right]=0_{1} \text { if } c_{i, j} \in \mathfrak{七}_{\alpha}^{h} \backslash\left\{a_{j}\right\} .
$$

3. Let $\psi_{3}=p_{m+1} \wedge \cdots \wedge p_{n}$ and observe that

$$
\psi_{3}\left[a_{m+1}, \ldots, a_{n}\right]=1 \text { and } \psi_{3}[\ldots, c, \ldots]<1 \text { for } c \neq 1 .
$$

By construction, $\psi=\psi_{1} \wedge \psi_{2} \wedge \psi_{3}$ satisfies our requirements.

Suppose now that $\mathbf{a}=\left(a_{1}, \ldots, a_{m}, a_{m+1}, \ldots, a_{k}, a_{k+1}, \ldots, a_{n}\right)$ (again allowing for some rearrangement of the elements in a) where

$$
a_{1}, \ldots, a_{m} \in \mathrm{七}_{\alpha}^{h} \backslash\{1\}, \quad a_{m+1}=\cdots=a_{k}=1, \quad \text { and } \quad a_{k+1}, \ldots, a_{n} \in \mathrm{七}_{\beta}^{h} \backslash\{1\} .
$$

We define a formula $\psi=\psi_{1} \vee \psi_{2} \vee \psi_{3} \vee \psi_{4} \vee \psi_{5}$ such that

$$
\psi[\mathbf{a}]<1 \quad \text { and } \quad \psi\left[\mathbf{c}_{i}\right]=1 \text { for all } i \in\{1, \ldots, n\} .
$$

1. Let $\psi_{1}=\neg \neg p_{1}^{r} \vee \cdots \vee \neg \neg p_{m}^{r}$ and observe that

$$
\psi_{1}\left[a_{1}, \ldots, a_{m}\right]=0_{1}<1 \quad \text { and } \quad \psi_{2}[\ldots, b, \ldots]=1 \text { for } b \in \mathfrak{七}_{\beta}^{h} .
$$

2. Let $\psi_{2}=\neg p_{m+1}^{r} \vee \cdots \vee \neg p_{n}^{r}$ and observe that

$$
\psi_{2}\left[a_{m+1}, \ldots, a_{n}\right]=0_{1}<1 \quad \text { and } \quad \psi_{2}[\ldots, b, \ldots]=1 \text { for } b \in \mathfrak{七}_{\alpha}^{h} \backslash\{1\} .
$$

3. Let $\psi_{3}=p_{k+1} \vee \cdots \vee p_{n}$ and observe that

$$
\psi_{3}\left[a_{k+1}, \ldots, a_{n}\right]<1 \text { and } \psi_{3}[\ldots, 1, \ldots]=1 .
$$


4. Using Theorem 3, for each $j \in\{1, \ldots, m\}$, there exists a propositional formula $\varphi_{j}\left(p_{j}\right)$ such that for all $i \in\{1, \ldots, n\}$,

$$
\varphi_{j}\left[a_{j}\right]=0_{1} \quad \text { and } \quad \varphi_{j}\left[c_{i, j}\right]=1 \text { if } c_{i, j} \in \mathrm{七}_{\alpha}^{h} \backslash\left\{a_{j}\right\} .
$$

Let $\psi_{4}=\varphi_{1} \vee \cdots \vee \varphi_{m}$. Then $\psi_{4}\left[a_{1}, \ldots, a_{m}\right]=0_{1}$ and for all $i \in\{1, \ldots, n\}$,

$$
\psi_{4}\left[c_{i, 1}, \ldots, c_{i, m}\right]=1 \text { if } c_{i, j} \in \mathrm{七}_{\alpha}^{h} \backslash\left\{a_{j}\right\} \text { for some } j \in\{1, \ldots, m\} .
$$

5. Let us temporarily interpret the constant $\perp$ by $0_{2}$. Then, using Theorem 3 , for each $j \in\{m+1, \ldots, n\}$, there exists a propositional formula $\varphi_{j}\left(p_{j}\right)$ such that, with respect to this temporary interpretation,

$$
\varphi_{j}\left[a_{j}\right]=0_{2}<1 \quad \text { and } \varphi_{j}\left[c_{i, j}\right]=1 \text { if } c_{i, j} \in \mathrm{七}_{\beta} \backslash\left\{a_{j}\right\} .
$$

Recall that $a_{k+1}^{r}=0_{2}$ and $c_{i, k+1}^{r}=0_{2}$ if $c_{i, k+1} \in \mathrm{七}_{\beta}^{h} \backslash\{1\}$. For each $j \in$ $\{m+1, \ldots, n\}$, let $\varphi_{j}^{\prime}$ be the formula obtained by replacing the constant symbol $\perp$ in $\varphi_{j}$ with the formula $p_{k+1}^{r}$ and observe that $\varphi_{j}^{\prime}\left[a_{j}, a_{k+1}\right]=0_{2}<1$ and

$$
\varphi_{j}^{\prime}\left[c_{i, j}, c_{i, k+1}\right]=1 \text { if } c_{i, j} \in \mathrm{七}_{\beta}^{h} \backslash\left\{a_{j}\right\} \text { and } c_{i, k+1} \in \mathrm{七}_{\beta}^{h} \backslash\{1\} .
$$

Now let $\psi_{5}=\varphi_{m+1}^{\prime} \vee \cdots \vee \varphi_{n}^{\prime}$ and observe that $\psi_{5}\left[a_{m+1}, \ldots, a_{n}\right]=0_{2}<1$ and $\psi_{5}\left[c_{i, m+1}, \ldots, c_{i, n}\right]=1$ if $c_{i, j} \in \mathrm{七}_{\beta}^{h} \backslash\left\{a_{j}\right\}$ and $c_{i, k+1} \in \mathrm{七}_{\beta}^{h} \backslash\{1\}$ for some $j \in\{m+1, \ldots, n\}$.

Finally, let $\psi=\psi_{1} \vee \psi_{2} \vee \psi_{3} \vee \psi_{4} \vee \psi_{5}$. Clearly $\psi[\mathbf{a}]<1$. To check that $\psi\left[\mathbf{c}_{i}\right]=1$ for all $i \in\{1, \ldots, n\}$, we consider the following cases:

1. If $c_{i, j} \in \mathrm{七}_{\beta}^{h}$ for some $j \in\{1, \ldots, m\}$, then $\psi\left[\mathbf{c}_{i}\right]=\psi_{1}\left[\mathbf{c}_{i}\right]=1$.

2. If $c_{i, j} \in \mathrm{E}_{\alpha}^{h} \backslash\{1\}$ for some $j \in\{m+1, \ldots, n\}$, then $\psi\left[\mathbf{c}_{i}\right]=\psi_{2}\left[\mathbf{c}_{i}\right]=1$.

3. If $c_{i, j}=1$ for some $j \in\{k+1, \ldots, n\}$, then $\psi\left[\mathbf{c}_{i}\right]=\psi_{3}\left[\mathbf{c}_{i}\right]=1$.

4. If $c_{i, j} \in \mathfrak{七}_{\alpha}^{h} \backslash\left\{a_{j}\right\}$ for some $j \in\{1, \ldots, m\}$, then $\psi\left[\mathbf{c}_{i}\right]=\psi_{4}\left[\mathbf{c}_{i}\right]=1$.

5. If $c_{i, j} \in \mathbf{E}_{\beta}^{h} \backslash\left\{a_{j}, 1\right\}$ for some $j \in\{m+1, \ldots, n\}$, then $\psi\left[\mathbf{c}_{i}\right]=\left(\psi_{3} \vee \psi_{5}\right)\left[\mathbf{c}_{i}\right]=$ 1.

Hence $\psi$ is an $\mathbf{a} / \mathbf{C}$ distinguishing formula.

Combining Lemmas 8 and 9 with the description of BL-chains provided in Theorem 4 , we obtain the following characterizations.

Theorem 5 The following are equivalent for any finite BL-chain $\mathbf{A}$ :

(1) The class of image-finite $\mathrm{K}(\mathbf{A})^{\mathrm{C}}$-models has the Hennessy-Milner property.

(2) The class of modally saturated $\mathrm{K}(\mathbf{A})^{\mathrm{C}}$-models has the Hennessy-Milner property.

(3) $\mathbf{A}$ is isomorphic to $\mathbf{L}_{\mathbf{n}+\mathbf{1}}$ or $\mathbf{L}_{\mathbf{n}+\mathbf{1}}^{\mathbf{h}} \oplus \mathbf{L}_{\mathbf{m}+\mathbf{1}}^{\mathbf{h}}$ for some $m, n \in \mathbb{N}$.

Theorem 6 For any standard BL-chain A:

(a) The class of image-finite $\mathrm{K}(\mathbf{A})^{\mathrm{C}}$-models has the Hennessy-Milner property if and only if $\mathbf{A}$ is isomorphic to either $\mathbf{L}_{\infty}$ or $\mathbf{L}_{\infty}^{\mathbf{h}} \oplus \mathbf{L}_{\infty}^{\mathbf{h}}$.

(b) The class of modally saturated $\mathrm{K}(\mathbf{A})^{\mathrm{C}}$-models does not have the Hennessy-Milner property. 


\section{Concluding Remarks}

In this paper, we have provided necessary and sufficient conditions on a chain-based algebra $\mathbf{A}$ for the classes of image-finite and modally saturated $\mathrm{K}(\mathbf{A})^{\mathrm{C}}$-models to have the Hennessy-Milner property, fully characterizing the case where $\mathbf{A}$ is a finite or standard BL-chain. Moreover, we expect that this approach can be extended in several directions. A treatment of alternative quantifiers (e.g., to express the average truth value at accessible worlds) or models based on (nonlinear) complete lattices might be obtained by suitably adapting the algebraic characterizations. We also aim to extend the approach beyond the crisp (Boolean-valued) setting to many-valued Kripke models where the accessibility relation is replaced by a binary map from states to elements of $\mathbf{A}$. This will require an appropriate definition of bisimulations between such models, perhaps following [12] where two different notions of a bisimulation are considered for many-valued modal logics based on Heyting algebras extended with additional constants for elements of the algebra.

Finally, let us remark that a promising methodology for tackling the extensions mentioned in the previous paragraph is provided by the recent paper [2], which presents a coalgebraic approach to expressivity in many-valued modal logics. The coalgebraic setting allows a wide range of many-valued modal logics, including those with crisp, many-valued, and probabilistic Kripke frames, to be defined uniformly by varying an underlying set endofunctor. By understanding modalities as many-valued predicate liftings, it is shown that a set of liftings satisfying a certain separation condition guarantees an expressive modal language for the logic. Indeed, the distinguishing formula property for image-finite models presented in Theorem 1 (first reported in [21]) follows as a direct corollary of this result. It is not yet clear how the strong distinguishing formula property for modally saturated models presented in Theorem 2 transfers to the coalgebraic setting, and characterization theorems such as Theorems 5 and 6 require a detailed algebraic study of the relevant structures. Nevertheless, the coalgebraic approach is, in our opinion, the most flexible and appropriate framework for further investigations into expressivity in many-valued modal logics.

\section{References}

1. P. Aglianò and F. Montagna. Varieties of BL-algebras I: General properties. Journal of Pure and Applied Algebra, 181:105-129, 2003.

2. M. Bílková and M. Dostal. Expressivity of many-valued modal logics, coalgebraically. In Proceedings of WoLLIC 2016, volume 9803 of LNCS, pages 109-124. Springer, 2016.

3. P. Blackburn, M. de Rijke, and Y. Venema. Modal logic. Cambridge University Press, 2001.

4. S. Borgwardt, F. Distel, and R. Peñaloza. The limits of decidability in fuzzy description logics with general concept inclusions. Artificial Intelligence, 218:23-55, 2015.

5. F. Bou, F. Esteva, L. Godo, and R. Rodríguez. On the minimum many-valued logic over a finite residuated lattice. Journal of Logic and Computation, 21(5):739-790, 2011.

6. X. Caicedo, G. Metcalfe, R. Rodríguez, and J. Rogger. Decidability in order-based modal logics. Journal of Computer System Sciences, 88:53-74, 2017.

7. X. Caicedo and R. Rodríguez. Standard Gödel modal logics. Studia Logica, 94(2):189-214, 2010.

8. X. Caicedo and R. Rodríguez. Bi-modal Gödel logic over [0,1]-valued Kripke frames. Journal of Logic and Computation, 25(1):37-55, 2015.

9. R. Cignoli, I. M. L. D’Ottaviano, and D. Mundici. Algebraic Foundations of Many-Valued Reasoning, volume 7 of Trends in Logic. Kluwer, Dordrecht, 1999. 
10. D. Diaconescu and G. Georgescu. Tense operators on MV-algebras and Łukasiewicz-Moisil algebras. Fundamenta Informaticae, 81(4):379-408, 2007.

11. D. Diaconescu, G. Metcalfe, and L. Schnüriger. A real-valued modal logic. In Proceedings of AiML 2016, pages 236-251. College Publications, 2016.

12. P. E. Eleftheriou, C. D. Koutras, and C. Nomikos. Notions of bisimulation for Heyting-valued modal languages. Journal of Logic and Computation, 22(2):213-235, 2012.

13. M. C. Fitting. Many-valued modal logics. Fundamenta Informaticae, 15(3-4):235-254, 1991.

14. M. C. Fitting. Many-valued modal logics II. Fundamenta Informaticae, 17:55-73, 1992.

15. L. Godo, P. Hájek, and F. Esteva. A fuzzy modal logic for belief functions. Fundamenta Informaticae, 57(2-4):127-146, 2003.

16. L. Godo and R. Rodríguez. A fuzzy modal logic for similarity reasoning. In Fuzzy Logic and Soft Computing, pages 33-48. Kluwer, 1999.

17. P. Hájek. Metamathematics of Fuzzy Logic. Kluwer, Dordrecht, 1998.

18. P. Hájek. Making fuzzy description logic more general. Fuzzy Sets and Systems, 154(1):1-15, 2005.

19. P. Hájek, D. Harmancová, and R. Verbrugge. A qualitative fuzzy possibilistic logic. International Journal of Approximate Reasoning, 12:1-19, 1995.

20. G. Hansoul and B. Teheux. Extending Łukasiewicz logics with a modality: Algebraic approach to relational semantics. Studia Logica, 101(3):505-545, 2013.

21. M. Marti and G. Metcalfe. Hennessy-Milner properties for many-valued modal logics. In Proceedings of AiML 2014, pages 407-420. College Publications, 2014.

22. R. McNaughton. A theorem about infinite-valued sentential logic. Journal of Symbolic Logic, 16(1):1-13, 1951 .

23. G. Metcalfe and N. Olivetti. Towards a proof theory of Gödel modal logics. Logical Methods in Computer Science, 7(2):1-27, 2011.

24. G. Metcalfe, N. Olivetti, and D. Gabbay. Proof Theory for Fuzzy Logics. Springer, 2008

25. G. Metcalfe, F. Paoli, and C. Tsinakis. Ordered algebras and logic. In H. Hosni and F. Montagna, editors, Uncertainty and Rationality, pages 1-85. Publications of the Scuola Normale Superiore di Pisa, Vol. 10, 2010.

26. G. Priest. Many-valued modal logics: a simple approach. Review of Symbolic Logic, 1:190-203, 2008.

27. S. Schockaert, M. De Cock, and E. Kerre. Spatial reasoning in a fuzzy region connection calculus. Artificial Intelligence, 173(2):258-298, 2009.

28. U. Straccia. Reasoning within fuzzy description logics. Journal of Artificial Intelligence Research, $14: 137-166,2001$

29. A. Vidal, L. Godo, and F. Esteva. On modal extensions of product fuzzy logic. Journal of Logic and Computation, 27(1): 299-336, 2017. 